\title{
ON A CONJECTURE ABOUT THE COMPARABILITY OF PARALLEL SYSTEMS WITH RESPECT TO THE CONVEX TRANSFORM ORDER*
}

\author{
BY
}

\author{
IDIR ARA B (COIMbRa), MILTO HAD J IKY R I A KOU (LARNAKA), AND \\ PAULO EDUARDO OLIVEIRA (COIMBRA)
}

\begin{abstract}
We study the comparability of the lifetimes of heterogeneous parallel systems with independent exponentially distributed components. It is known that the order statistics of systems composed of two types of components may be comparable with respect to the star transform order. On what concerns the stronger convex transform order, results have been obtained only for the sample maxima assuming that one of the systems is homogeneous. We prove, under the same assumptions as for the star transform ordering, that the lifetimes of heterogeneous parallel systems are not comparable with respect to the convex transform order.
\end{abstract}

2020 Mathematics Subject Classification: Primary 60E15; Secondary $60 \mathrm{E} 05,62 \mathrm{~N} 05$.

Key words and phrases: star transform order, convex transform order, failure rate, sign variation.

\section{INTRODUCTION}

Deciding about the ageing properties of systems whose lifetime is random requires an appropriate meaning of the comparison criterion. The literature is abundant in alternative definitions of ageing properties and the corresponding orderings. Generally speaking, the approach starts by defining how to measure the relevant risk, which will be considered as the failure rate. The characterization of the monotonicity of the failure rate function for a lifetime distribution is an important aspect and has been studied, among others, by Barlow and Proschan [4], Patel [16], Sengupta [17] or El-Bassiouny [7].

Once we define a risk measure, we may become interested in ordering lifetime distributions with respect to the risk considered. This may be viewed as deciding

* This work was partially supported by the Centre for Mathematics of the University of Coimbra - UIDB/00324/2020, funded by the Portuguese Government through FCT/MCTES. 
which distribution is ageing faster. Order relations, mostly falling into some family of transform orders, have been studied extensively by various authors (see, for example, Desphande et al. [6], Kochar and Wiens [10], Singh [19], Fagiuoli and Pellerey [8] or Shaked and Shanthikumar [18]). We refer the reader to Shaked and Shanthikumar [18, Section 4.B] for general definitions and properties of transform order relations. We mention that the convex transform order that we will be considering later has a geometric interpretation, as described by van Zwet [21], providing a way to compare the skewness of lifetime distributions.

The order properties of the lifetime distributions of parallel systems have been thoroughly studied in the literature, with some recent results in Zhang et al. [23], Kayal [9] or Wu et al. [22], where the role of different lifetime distribution of the components is studied, and in Cai et al. [5] or $\mathrm{Li}$ and $\mathrm{Li}$ [13] dealing with the effect of dependence of component lifetimes.

The present note responds to a question raised by Kochar and Xu [11]. These authors were interested in comparing the ageing performance of parallel systems with respect to the convex transform order when the components have exponential and independent lifetime distributions. Kochar and Xu [11, Theorem 3.1] proved that, for systems with the same number of components, a parallel system with homogeneous components ages faster than a parallel system with heterogeneous components. Kochar and Xu [11, Remark 3.2] conjectured that the same ageing behaviour holds when comparing two heterogeneous systems based on components that have exponentially distributed lifetimes with hazard rates that can be ordered in a suitable way (see Definition 3.1 below for details). Their conjecture is based on the intuitive extension of their Theorem 3.1 and on numerical evidence they collected. Although Kochar and Xu [12] proved that the ordering relationship holds with respect to the star transform order under an alternative formulation of the assumptions on the parameters, the corresponding result for the convex transform order remained open.

In this paper we give a simpler proof of the star ordering proved in [12], based on quite different arguments. Our approach to the proof of the star transform ordering provides a method allowing us to show that the Kochar and Xu conjecture on the convex transform order is not valid. Furthermore, it is important to highlight that the contribution of this work is not only the disproval of the conjecture (this could have been obtained via a counterexample) but also the fact that our proof gives a general method to obtain regions where the required relationship defining the convex order fails. These regions seem to be rather narrow, so some insight into their location is significant to decide about the ordering.

\section{PRELIMINARIES}

Let $X$ be a nonnegative random variable with density function $f_{X}$, distribution function $F_{X}$, and tail function $\bar{F}_{X}=1-F_{X}$. Moreover, for each $x \geqslant 0$ the failure 
rate function of $X$ is given by $r_{X}=f_{X} / \bar{F}_{X}$. Two of the most simple and common ageing notions are defined in terms of the failure rate function.

DEFINITION 2.1. Let $X$ be a nonnegative valued random variable.

- $X$ is IFR (resp., DFR) if $r_{X}$ is increasing (resp., decreasing) for $x \geqslant 0$.

- $X$ is IFRA (resp., DFRA) if $\frac{1}{x} \int_{0}^{x} r_{X, s}(t) d t$ is increasing (resp., decreasing) for $x>0$.

The above definitions refer to monotonicity properties of the distribution. In the following, we introduce criteria to compare distribution functions.

DEFINITION 2.2. Let $\mathcal{F}$ denote the family of distribution functions such that $F(0)=0$, and $X$ and $Y$ be nonnegative random variables with distribution functions $F_{X}, F_{Y} \in \mathcal{F}$.

The random variable $X$ (or its distribution $F_{X}$ ) is said to be smaller than $Y$ (or its distribution $F_{Y}$ ) in convex transform order, and we write $X \leqslant_{\mathrm{c}} Y$, or $F_{X} \leqslant_{\mathrm{c}} F_{Y}$, if $\bar{F}_{Y}^{-1}\left(\bar{F}_{X}(x)\right)$ is convex.

The random variable $X$ (or its distribution $F_{X}$ ) is said to be smaller than $Y$ (or its distribution $F_{Y}$ ) in star transform order, and we write $X \leqslant_{*} Y$, or $F_{X} \leqslant_{*} F_{Y}$, if $\frac{\bar{F}_{Y}^{-1}\left(\bar{F}_{X}(x)\right)}{x}$ is increasing (this is also known as $\bar{F}_{Y}^{-1}\left(\bar{F}_{X}(x)\right)$ being star-shaped).

The definitions above fall into the family of iterated IFR and IFRA orders, respectively, introduced and initially studied by Nanda et al. [15], Arab and Oliveira [1], [2] or Arab et al. [3], considering their iteration parameter to be 1. It is particularly useful to highlight at this point that the IFR and IFRA orders are scale invariant (see Nanda et al. [15]). Consequently, in the case of families of distributions that have scale parameters, this allows one to choose them in the most convenient way.

A general characterization of transform order relations is given below.

Theorem 2.1 (Nanda et al. [15, Propositions 3.1 and 4.1]). Let $X$ and $Y$ be random variables with distribution functions $F_{X}, F_{Y} \in \mathcal{F}$.

(1) $X \leqslant_{*} Y$ if and only if for any real number $a, \bar{F}_{Y}(x)-\bar{F}_{X}(a x)$ changes sign at most once, and if the change of sign occurs, it is in the order "-, +" as $x$ varies from 0 to $+\infty$.

(2) $X \leqslant_{\mathrm{c}} Y$ if and only if for any real numbers $a$ and $b, \bar{F}_{Y}(x)-\bar{F}_{X}(a x+b)$ changes sign at most twice, and if the change of sign occurs twice, it is in the order “,,+-+ ” as $x$ varies from 0 to $+\infty$.

REMARK 2.1. As mentioned by Arab and Oliveira [1, Remark 25], it is enough to verify the above characterizations for $a>0$. Moreover, when describing a sign variation of any given function, we will always be considering that $x$ goes from 0 to $+\infty$. 
The characterization given by Theorem 2.1 requires explicit expressions of the tails of the distributions, which are often unavailable. Computationally tractable characterizations were studied by Arab and Oliveira [1], [2] and Arab et al. [3, Theorems 2.3 and 2.4]. As one may see in the proofs in [1], the control of sign variation is usually more complex when $b<0$. However, a prior verification of the star transform ordering will help circumvent this difficulty.

THEOREM 2.2 (Arab et al. [3, Theorem 29]). Let $X$ and $Y$ be random variables with distribution functions $F_{X}, F_{Y} \in \mathcal{F}$. If $X \leqslant_{*} Y$ and the criterion in Theorem 2.1 (2) is satisfied for $b \geqslant 0$, then $X \leqslant_{c} Y$.

The lifetime of parallel systems is expressed as the maximum of the lifetimes of the components. When these are exponentially distributed, the distribution function of the system lifetime is a linear combination of exponential terms. Later, we will be interested in counting and localizing the roots of such expressions. The following result will play an important role.

THEOREM 2.3 (Tossavainen [20]). Let $n \geqslant 0, p_{0}>p_{1}>\cdots>p_{n}>0$, and $\alpha_{j} \neq 0, j=0,1, \ldots, n$, be real numbers. Then the function $f(t)=\sum_{j=0}^{n} \alpha_{j} p_{j}^{t}$ has no real zeros if $n=0$, and for $n \geqslant 1$ it has at most as many real zeros as there are sign changes in the sequence $\alpha_{0}, \alpha_{1}, \ldots, \alpha_{n}$.

\section{MAIN RESULTS}

We begin this section by quoting a result by Kochar and $\mathrm{Xu}$ [11] that suggested the conjecture we will be discussing later and therefore it is presented here for the sake of completeness.

Theorem 3.1 (Kochar and Xu [11, Theorem 3.1]). Let $X_{1}, \ldots, X_{n}$ be independent and exponentially distributed random variables with common hazard rate $\lambda$. Similarly, let $Y_{1}, \ldots, Y_{n}$ be independent and exponentially distributed random variables with hazard rates $\theta_{i}, i=1, \ldots, n$. Then $\max \left(X_{1}, \ldots, X_{n}\right) \leqslant_{\mathrm{c}}$ $\max \left(Y_{1}, \ldots, Y_{n}\right)$.

For the remainder of this section, we will be interested in characterizing the order relationship between parallel systems of heterogeneous components with exponential lifetime distributions. We recall an order relation between $\mathbb{R}^{n}$ vectors introduced by Marshall and Olkin [14, Definition A.1].

Definition 3.1. Let $\left(\lambda_{1}, \ldots, \lambda_{n}\right),\left(\theta_{1}, \ldots, \theta_{n}\right) \in \mathbb{R}^{n}$. Denote by $\lambda_{(1)} \leqslant$ $\cdots \leqslant \lambda_{(n)}$ the ordered coordinates of the first vector, and likewise for the second vector. We write $\left(\lambda_{1}, \ldots, \lambda_{n}\right) \prec\left(\theta_{1}, \ldots, \theta_{n}\right)$ if

$$
\sum_{i=1}^{k} \lambda_{(i)} \geqslant \sum_{i=1}^{k} \theta_{(i)} \quad \text { for } k=1, \ldots, n-1, \quad \text { and } \quad \sum_{i=1}^{n} \lambda_{(i)}=\sum_{i=1}^{n} \theta_{(i)} .
$$


REMARK 3.1. Without loss of generality, we can assume the components of the hazard rate vectors $\left(\lambda_{1}, \ldots, \lambda_{n}\right)$ are arranged increasingly, therefore in what follows, we will be assuming our hazard rate vectors satisfy $\lambda_{k} \leqslant \lambda_{m}$ when $k \leqslant m$.

Based on their proof and some numerical evidence, Kochar and $\mathrm{Xu}$ [11] conjectured that the conclusion of Theorem 3.1 still holds if the $X_{i}$ have hazard rates $\lambda_{i}$ and the $Y_{i}$ have hazard rates $\theta_{i}$ such that $\left(\lambda_{1}, \ldots, \lambda_{n}\right) \prec\left(\theta_{1}, \ldots, \theta_{n}\right)$.

3.1. The star transform ordering. Kochar and $\mathrm{Xu}$ [12] proved a stronger ordering result but with respect to the weaker star transform order.

Theorem 3.2 (Kochar and Xu [12, Theorem 3.1]). Let $X_{1}, \ldots, X_{n}$ be independent and exponentially distributed where $X_{1}, \ldots, X_{p}$ have hazard rate $\lambda_{1}$ and $X_{p+1}, \ldots, X_{n}$ have hazard rate $\lambda_{2}$, and let $Y_{1}, \ldots, Y_{n}$ satisfy similar conditions with hazard rates $\theta_{1}$ and $\theta_{2}$. If $\lambda_{2} / \lambda_{1} \geqslant \theta_{2} / \theta_{1}$, then the $k$-th order statistics are ordered with respect to the star transform order, that is, $X_{k: n} \leqslant_{*} Y_{k: n}$.

REMARK 3.2. The star transform order is insensitive to multiplication of the random variables by constants. Taking this into account, it is easily seen that an equivalent formulation of Theorem 3.2 is obtained by assuming that the hazard rates satisfy $\left(\lambda_{1}, \lambda_{2}\right) \prec\left(\theta_{1}, \theta_{2}\right)$.

In Subsection 3.2, we will compare the lifetimes of parallel systems with respect to the convex transform order. Our approach is based on characterizing the sign variation of suitable functions, as expressed by Theorem 2.1. Therefore, taking into account Theorem 2.2, the following reduced version of Theorem 3.2 provides a useful first step for the proof of Theorem 3.4, and provides some calculation details.

THEOREM 3.3. Let $X_{1}$ and $X_{2}$ be independent and exponentially distributed with hazard rates $\lambda_{1}$ and $\lambda_{2}$, respectively. Analogously, let $Y_{1}$ and $Y_{2}$ be independent and exponentially distributed with hazard rates $\theta_{1}$ and $\theta_{2}$, respectively. If $\left(\lambda_{1}, \lambda_{2}\right) \prec\left(\theta_{1}, \theta_{2}\right)$, then $X=\max \left(X_{1}, X_{2}\right) \leqslant * Y=\max \left(Y_{1}, Y_{2}\right)$.

Proof. If $\lambda_{1}=\theta_{1}$, Definition 3.1 implies that $\left(\lambda_{1}, \lambda_{2}\right)=\left(\theta_{1}, \theta_{2}\right)$, and $X$ and $Y$ are equivalent. Suppose now that $\lambda_{1}>\theta_{1}$. Let $\bar{F}_{X}$ and $\bar{F}_{Y}$ be the survival functions of $X$ and $Y$. Then

$$
\begin{aligned}
& \bar{F}_{X}(x)=e^{-\lambda_{1} x}+e^{-\lambda_{2} x}-e^{-\left(\lambda_{1}+\lambda_{2}\right) x}, \\
& \bar{F}_{Y}(x)=e^{-\theta_{1} x}+e^{-\theta_{2} x}-e^{-\left(\theta_{1}+\theta_{2}\right) x} .
\end{aligned}
$$

Taking into account Theorem 2.1 and Remark 2.1, it is sufficient to prove that $\bar{V}(x)=\bar{F}_{Y}(x)-\bar{F}_{X}(a x)$ changes sign at most once, and if the sign change occurs, it is in the order ",-+ " for every real $a>0$. We will consider three separate cases, depending on the value of $a$. First, note that the assumptions on the hazard rates imply that $\theta_{1}<\lambda_{1}<\lambda_{2}<\theta_{2}$ and $\lambda_{1}+\lambda_{2}=\theta_{1}+\theta_{2}$, hence neither system has homogeneous components. 
CASE 1: $a=1$. We have $\bar{V}(x)=e^{-\theta_{1} x}+e^{-\theta_{2} x}-\left(e^{-\lambda_{1} x}+e^{-\lambda_{2} x}\right)$. Reordering the exponential terms so that they appear in decreasing order of their basis, the sign pattern of the coefficients is ",,,+--+ ". Hence, according to Theo$\operatorname{rem} 2.3, \bar{V}$ has at most two real roots. Moreover, $\lim _{x \rightarrow-\infty} \bar{V}(x)=+\infty$, while $\lim _{x \rightarrow+\infty} \bar{V}(x)=0^{+}$. Furthermore, taking into account that $\bar{V}(0)=0, \bar{V}^{\prime}(0)=0$ and $\bar{V}^{\prime \prime}(0)=-\lambda_{1}^{2}+\theta_{1}^{2}-\lambda_{2}^{2}+\theta_{2}^{2}=-\left(\theta_{2}-\lambda_{2}\right)\left(\lambda_{1}-\lambda_{2}+\theta_{1}-\theta_{2}\right)>0$, it follows that $\bar{V}(x) \geqslant 0$, which means that $\bar{F}_{Y}(x) \geqslant \bar{F}_{X}(x)$ for every $x \in \mathbb{R}$, thus no sign changes occur.

CASE 2: $a>1$. As $\bar{F}_{X}$ is decreasing, it follows that, for $x \geqslant 0, \bar{V}(x) \geqslant$ $\bar{F}_{Y}(x)-\bar{F}_{X}(x) \geqslant 0$, so again no sign changes occur.

CASE 3: $0<a<1$. To analyse the sign pattern of the coefficients in $\bar{V}$, we distinguish two cases:

$\theta_{1}<a \lambda_{1}$ : After reordering the exponentials in $\bar{V}$ according to their basis, the sign pattern of the coefficients is “,,,,,+--++- ". Thus, according to Theorem 2.3, $\bar{V}$ has at most three real roots. The sign pattern of the coefficients implies that $\lim _{x \rightarrow-\infty} \bar{V}(x)=-\infty$, while $\lim _{x \rightarrow+\infty} \bar{V}(x)=0^{+}$. Finally, taking into account that $\bar{V}(0)=\bar{V}^{\prime}(0)=0$, the possible sign changes for $\bar{V}$ are either "+" or ",-+ ".

$\theta_{1} \geqslant a \lambda_{1}:$ As $\bar{F}_{X}$ is decreasing and $a \leqslant \theta_{1} / \lambda_{1}$, it follows that, for $x \geqslant 0$,

$$
\begin{aligned}
\bar{V}(x) & \leqslant \bar{H}(x)=\bar{F}_{Y}(x)-\bar{F}_{X}\left(\frac{\theta_{1}}{\lambda_{1}} x\right) \\
& =e^{-\theta_{2} x}-e^{-\left(\theta_{1}+\theta_{2}\right) x}-\left(e^{-\frac{\theta_{1} \lambda_{2}}{\lambda_{1}} x}-e^{-\frac{\theta_{1}}{\lambda_{1}}\left(\lambda_{1}+\lambda_{2}\right) x}\right) .
\end{aligned}
$$

After reordering the exponentials in $\bar{H}$ according to their basis, the sign pattern of the coefficients in $\bar{H}$ is ",,,-++- ", implying that, according to Theorem 2.3 . $\bar{H}$ has at most two real roots. The sign of the coefficients of $\bar{H}$ also implies that $\lim _{x \rightarrow+\infty} \bar{H}(x)=0^{-}$, which, together with the fact that $\bar{H}(0)=\bar{H}^{\prime}(0)=0$ and $\bar{H}^{\prime \prime}(0)=2 \theta_{1}\left(\frac{\theta_{1}}{\lambda_{1}} \lambda_{2}-\theta_{2}\right)<0$, further implies that $\bar{H}(x) \leqslant 0$, so $\bar{V}(x) \leqslant 0$, that is, no sign changes occur.

So, finally, $\bar{V}$ has at most one sign change when $x$ goes from 0 to $+\infty$, and if the change occurs, it is in the order ",-+ ". Thus, according to Theorem 2.1 , $X \leqslant{ }_{*} Y$.

Recalling Remark 3.2, the following alternative equivalent formulation is immediate.

COROllary 3.1. Let $X_{1}$ and $X_{2}$ be independent and exponentially distributed with hazard rates $\lambda_{1}$ and $\lambda_{2}$, respectively. Analogously, let $Y_{1}$ and $Y_{2}$ be independent and exponentially distributed with hazard rates $\theta_{1}$ and $\theta_{2}$, respectively. If $\lambda_{2} / \lambda_{1} \geqslant \theta_{2} / \theta_{1}$, then $X=\max \left(X_{1}, X_{2}\right) \leqslant * Y=\max \left(Y_{1}, Y_{2}\right)$. 
REMARK 3.3. Note that although Theorem 3.2 allows for an arbitrary number of components, each system is only allowed to have two different types of component. On the other hand, Theorem 3.3 is obtained for lifetimes which may all have different hazard rates, but its proof is limited to systems with only two components. It remains an open problem to prove the star transform order between systems with more than three types of components.

3.2. The convex transform ordering. We may now give a partial answer to the conjecture announced by Kochar and $\mathrm{Xu}$ [11], about the comparability of the lifetime distributions of parallel systems with respect to the convex transform order.

THEOREM 3.4. Let $X$ and $Y$ be as in Theorem 3.3. Then $X$ and $Y$ are not comparable with respect to the convex transform order.

Proof. As before, if $\lambda_{1}=\theta_{1}$, then $X$ and $Y$ are equivalent. Suppose now that $\lambda_{1}>\theta_{1}$. We start by noticing that the sign variation analysis of $\bar{V}(x)=$ $\bar{F}_{Y}(x)-\bar{F}_{X}(a x+b)$ is inconclusive. Note that, unlike in the star transform order case (Theorem 3.3, we have $\bar{V}(0)=1-\bar{F}_{X}(b)>0$.

The favourable cases. Taking into account Theorems 2.1 and 2.2 , and $\mathrm{Re}-$ mark 2.1, we need to describe the sign variation of $\bar{V}$ for $a, b>0$. In each case, control is obtained by identifying the possible number of real roots with the use of Theorem 2.3, and coupling this with the behaviour of $\bar{V}$ when $x \rightarrow \pm \infty$.

CASE $1: a \geqslant 1$. As $b>0$ and $x \geqslant 0$, we have $a x+b \geqslant x$, hence $\bar{V}(x) \geqslant$ $\bar{F}_{Y}(x)-\bar{F}_{X}(x) \geqslant 0$.

CASE 2: $\theta_{1} / \lambda_{1} \leqslant a<1$. Reordering the terms in $\bar{V}$ appropriately, the sign pattern of its coefficients is ",,,,,+--++- " ( or “,,,,+-++- " if $\left.a=\theta_{1} / \lambda_{1}\right)$, hence, according to Theorem 2.3, $\bar{V}$ has at most three real roots. Moreover, it is easily seen that $\lim _{x \rightarrow+\infty} \bar{V}(x)=0^{+}$. As $\bar{V}(0)>0$, this means there are at most two nonnegative real roots. Thus, the sign pattern can only be either "+" or "+,,-+ ".

CASE 3: $0<a \leqslant \theta_{1} / \lambda_{2}<1$. Reordering again the terms in $\bar{V}$ to apply Theorem 2.3, we find the sign pattern for its coefficients is “-,,,,,-+++- " (collapsing to "-,,,,+++- " if $a=\theta_{1} / \lambda_{2}$ ), so $\bar{V}$ has at most two real roots. At infinity, we find that $\lim _{x \rightarrow+\infty} \bar{V}(x)=0^{-}$. Hence, as $\bar{V}(0)>0, \bar{V}$ has one nonnegative real root and the sign pattern of $\bar{V}$ is “+, -".

The violating case: $\theta_{1} / \lambda_{2}<a<\theta_{1} / \lambda_{1}$. The sign pattern of the ordered coefficients in $\bar{V}$ is ",,,,,-+-++- ". Thus, using Theorem 2.3, $\bar{V}$ may have up to four real roots. The fact that $\lim _{x \rightarrow-\infty} \bar{V}(x)=-\infty$, together with $\bar{V}(0)>0$, implies that one of the roots is negative. Moreover, $\lim _{x \rightarrow+\infty} \bar{V}(x)=0^{-}$, so this is compatible with the sign variations (when $x$ varies from 0 to $+\infty$ ) ",+- , or ",,,+-+- ". That is, the use of Theorem 2.3 is not conclusive. 
We need to show that the sign variation ",,,+-+- " is indeed achieved for an appropriate choice of $a, b>0$, hence violating the comparison criterion. From the previous analysis, if $a=\theta_{1} / \lambda_{2}$ the sign variation of $\bar{V}$ is ",+- ". Likewise, if $a=\theta_{1} / \lambda_{1}$ the sign variation of $\bar{V}$ is either " + " or ",,+-+ ". Choose $b_{0}>0$ so that the sign variation of $\bar{V}(x)=\bar{F}_{Y}(x)-\bar{F}_{X}\left(\frac{\theta_{1}}{\lambda_{1}} x+b_{0}\right)$ is “+, -, +", and keep this choice fixed for the rest of the proof. Furthermore, remember that when $\theta_{1} / \lambda_{2}<a<\theta_{1} / \lambda_{1}$, we have verified that $\lim _{x \rightarrow+\infty} \bar{V}(x)=0^{-}$. Hence we have the following graphical description of the sign of $\bar{V}$, depending on $x$ and $a$ :

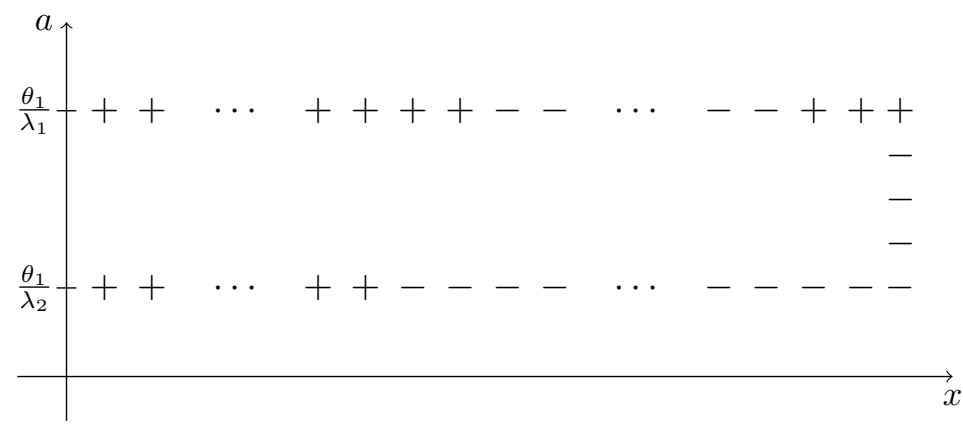

Keeping $b_{0}$ fixed, we may look at $\bar{V}$ as a function of $x$ and $a$. Thus, we may differentiate with respect to $a$, to find

$$
\frac{\partial \bar{V}}{\partial a}(x, a, b)=x f_{X}(a x+b),
$$

where $f_{X}$ is the density function of $X$, implying that for every possible choice for $b>0$, in particular for $b=b_{0}, \frac{\partial \bar{V}}{\partial a}(x, a, b)>0$. Hence, as a function of $a$ alone, $\bar{V}$ is increasing. Thus, when $a$ increases from $\theta_{1} / \lambda_{2}$ to $\theta_{1} / \lambda_{1}$ the value for $\bar{V}$ is also increasing, so once it becomes positive it may no longer get back to negative values. For the particular choice $b=b_{0}$ which produces the lines of signs "+" and "-" above, the increase of $\bar{V}$ with respect to $a$ explains why the initial sequence of " + " signs for $a_{0}=\theta_{1} / \lambda_{1}$ is longer than the corresponding initial sequence when $a=\theta_{1} / \lambda_{2}$.

It remains to justify that the choice for $b_{0}$ does exist. First, note that we proved in Case 3 of the proof of Theorem 3.3 that $\bar{F}_{Y}(x)-\bar{F}_{X}\left(a_{0} x\right) \leqslant 0$ for every $x \geqslant 0$, and the inequality is strict for every $x>0$. Now, choosing some $x_{0}>0$, we have $\bar{F}_{X}^{-1}\left(\bar{F}_{Y}\left(x_{0}\right)\right)>a_{0} x_{0}$, so we may find $b_{0}$ (depending on $x_{0}$ ) such that $\bar{F}_{X}^{-1}\left(\bar{F}_{Y}\left(x_{0}\right)\right)>a_{0} x_{0}+b_{0}$, which implies that $\bar{F}_{Y}\left(x_{0}\right)<\bar{F}_{X}\left(a_{0} x_{0}+b_{0}\right)$. As the functions are continuous, this inequality will hold on some neighbourhood of $x_{0}$, so the sign pattern represented above always happens.

Getting back to the graphical representation above, we now locate the set of points $(x, a)$ such that $\bar{V}\left(x, a, b_{0}\right)=0$, that is, we may take $a=h(x)$. As $\bar{V}$ is 
continuous, this function $h$ is also continuous, and we will find the behaviour of $h$ described by the thick line below, where we also identify the sign of $\bar{V}$ in each region:

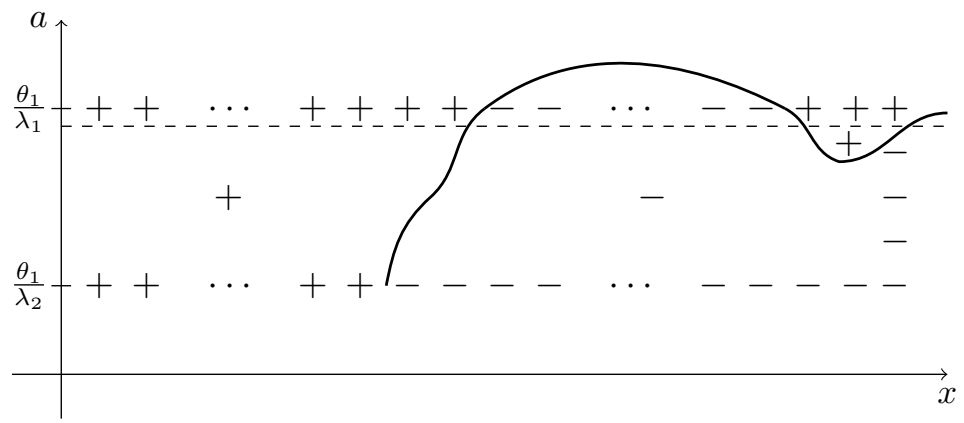

The position of the horizontal dashed line identifies a value of the parameter $a$ for which the sign variation of $\bar{V}$, with $b=b_{0}$, is actually ",,,+-+- ", so the random variables are not comparable with respect to the convex transform order.

REMARK 3.4. Although a counterexample would have been enough, the proof of Theorem 3.4 goes one step further, as it shows how to identify a set of parameters for which the convex transform order comparability fails, that Kochar and Xu [11] could not locate, since without prior indication of where to look for, it is easy to miss the appropriate choice for $a$ and $b$. The construction above depends critically on an appropriate choice for $b$, producing the ",,+-+ " sign pattern on the top line. Numerical experiments confirm the arguments produced in the course of the proof, indicating that $b$ must be chosen close to 0 . Moreover, experiments suggest that even with a convenient choice of $b$ the top-right region with the " + " signs may be relatively narrow.

The original motivation for the conjecture stems from the characterization of skewness of the densities, in the sense introduced by van Zwet [21], which was the problem studied by Kochar and Xu [11]. The link between the convexity approach used by Kochar and $\mathrm{Xu}$ [11] and our sign variation approach follows from the characterization of convexity by means of sign variation, described by Arab and Oliveira [1, Theorem 20], [2]. The narrow region for the choice of $a$ and $b$ violating the sign variation pattern means that the function $\bar{F}_{Y}^{-1}\left(\bar{F}_{X}(x)\right)$ is concave in almost the whole of its domain and convex in a small interval.

An explicit example and choice of the parameters violating the $\leqslant_{c}$-comparability may be obtained by taking, for example, $\lambda_{1}=2, \lambda_{2}=3, \theta_{1}=1.5, \theta_{2}=3.5$, $a=0.749$ and $b=0.0125$. Note that the choice of hazard rates and parameters is meant to identify where the sign variation condition is violated, and not directly the convexity of $\bar{F}_{Y}^{-1}\left(\bar{F}_{X}(x)\right)$ itself. The graphical representation of $\bar{F}_{Y}^{-1}\left(\bar{F}_{X}(x)\right)$ is not very illustrative, as this function behaves almost as a straight line. Moreover, the function $\bar{F}_{Y}$ is obviously not explicitly invertible, so simple closed rep- 
resentations of the derivatives seem to be out of reach. However, proceeding numerically, one may obtain the approximations $\bar{F}_{Y}^{-1}\left(\bar{F}_{X}\right)^{\prime \prime}(1.5) \approx 0.20344$ and $\bar{F}_{Y}^{-1}\left(\bar{F}_{X}\right)^{\prime \prime}(2.5) \approx-0.06557$, hence the function is neither convex nor concave.

Let us go back to the framework of Theorems 3.3 and 3.4 and describe the sign pattern of $\bar{V}(x, a, b)=\bar{F}_{Y}(x)-\bar{F}_{X}(a x+b)$, considering the effect of the three variables $x, a$ and $b$. It is easily seen that the intersection of the surface defined by $\bar{V}(x, a, b)=0$ with the plane $b=0$ is described by $a=\frac{\bar{F}_{X}^{-1}\left(\bar{F}_{Y}(x)\right)}{x}$, and the intersection with the plane $a=0$ by $b=\bar{F}_{X}^{-1}\left(\bar{F}_{Y}(x)\right)$. Therefore, the intersection with the plane $b=0$ defines, as a function of $x$, a decreasing curve. An inspection of the proof of Theorem 3.3 implies that this function is always $\geqslant \theta_{1} / \lambda_{1}$. Indeed, the proof of Theorem 3.3 shows that for $(x, a, 0)$ with $x \geqslant 0$ and $a \leqslant \theta_{1} / \lambda_{1}$ the sign of $\bar{V}$ is negative, hence this region does not intersect the surface $\bar{V}(x, a, b)=0$. On the other hand, when $x \geqslant 0$ and $a>\theta_{1} / \lambda_{1}$, the sign pattern of $\bar{V}(x, a, 0)$ may be "-, +", therefore an intersection with $\bar{V}(x, a, b)=0$ may occur. These considerations imply that the curve $a(x)=\frac{\bar{F}_{X}^{-1}\left(\bar{F}_{Y}(x)\right)}{x}$ is decreasing and has a lower bound. As this function is obviously nonnegative, $\lim _{x \rightarrow+\infty} a(x)$ is finite and is $\geqslant \theta_{1} / \lambda_{1}$. Moreover, taking into consideration that the sign variation of $\bar{F}_{X}^{-1}\left(\bar{F}_{Y}(x)\right)-\left(\frac{\theta_{1}}{\lambda_{1}} x+b\right)$ is either "-" or "-, +, -", it follows that $\lim _{x \rightarrow+\infty} \bar{F}_{X}^{-1}\left(\bar{F}_{Y}(x)\right)-\frac{\theta_{1}}{\lambda_{1}} x=0$. Therefore, for the particular example mentioned above, where $\left(\lambda_{1}, \lambda_{2}\right)=(2,3)$ and $\left(\theta_{1}, \theta_{2}\right)=(1.5,3.5)$, we have $\lim _{x \rightarrow+\infty} \bar{F}_{X}^{-1}\left(\bar{F}_{Y}(x)\right)-\frac{3}{4} x=0$.

We now address an extension of Theorem 3.4 to a generalized version of the framework of Theorem 3.2. The observation above that $\bar{F}_{Y}^{-1}\left(\bar{F}_{X}(x)\right)$ behaves almost like a straight line suggests a criterion for noncomparability, which we apply to lifetimes of parallel systems.

THEOREM 3.5. Let $X_{1}, \ldots, X_{n}$ be independent and exponentially distributed random variables where $X_{1}, \ldots, X_{p}$ have hazard rate $\lambda_{1}$ and $X_{p+1}, \ldots, X_{n}$ have hazard rate $\lambda_{2}$, and let $Y_{1}, \ldots, Y_{n}$ satisfy similar conditions with hazard rates $\theta_{1}$ and $\theta_{2}$. If $\left(\lambda_{1}, \lambda_{2}\right) \prec\left(\theta_{1}, \theta_{2}\right)$, then $X=\max \left(X_{1}, \ldots, X_{n}\right)$ and $Y=\max \left(Y_{1}, \ldots, Y_{n}\right)$ are not comparable with respect to the convex transform order.

Proof. We seek to conclude that $\bar{F}_{Y}^{-1}\left(\bar{F}_{X}(x)\right)$ is neither convex nor concave. It is easily seen that this function is increasing and $\bar{F}_{Y}^{-1}\left(\bar{F}_{X}(0)\right)=0$. We shall prove that, as $x \rightarrow+\infty, \bar{F}_{Y}^{-1}\left(\bar{F}_{X}(x)\right)$ approaches a straight line that starts at the origin. Hence, nonconvexity follows immediately. Before proceeding, notice that $\bar{F}_{Y}^{-1}\left(\bar{F}_{X}(x)\right)=F_{Y}^{-1}\left(F_{X}(x)\right), F_{Y}(x)=F_{1}^{p}(x) F_{2}^{n-p}(x)$ and $F_{X}(x)=$ $G_{1}^{p}(x) G_{2}^{n-p}(x)$, where $F_{i}(x)=1-e^{-\theta_{i} x}$ and $G_{i}(x)=1-e^{-\lambda_{i} x}$. Taking into account the comments above, choose $c_{1}=\lambda_{1} / \theta_{1}>1$ and consider $H(x)=$ $F_{X}(x)-F_{Y}\left(c_{1} x\right)$. After expansion, $H(x)$ is represented as a linear combination 
of exponentials. Moreover, when $x \rightarrow+\infty$, the dominant terms correspond to the smallest values of the multiplicative constants appearing in the exponents. That is, considering the choice for $c_{1}$, the dominant terms are the ones that involve $e^{-\lambda_{1} x}$. Consider now the perturbation $H_{1}(x)=F_{X}(x)-F_{Y}\left(c_{1} x-\varepsilon\right)$, where $\varepsilon>0$. It is obvious that $\lim _{x \rightarrow+\infty} H_{1}(x)=0$ and $H_{1}^{\prime}(x)=f_{X}(x)-c_{1} f_{Y}\left(c_{1} x-\varepsilon\right)$. The dominant term, when $x \rightarrow+\infty$, in $H_{1}^{\prime}(x)$ is $\lambda_{1} e^{-\lambda_{1} x}\left(1-e^{\theta_{1} \varepsilon}\right)<0$. Hence, for large values of $x, H_{1}$ is decreasing to 0 , that is, $F_{X}(x) \geqslant F_{Y}\left(c_{1} x-\varepsilon\right)$. Likewise, for $x$ large enough, $F_{X}(x) \leqslant F_{Y}\left(c_{1} x+\varepsilon\right)$. As $F_{Y}$ is increasing, these two inequalities imply that, when $x \rightarrow+\infty,\left|F_{Y}^{-1}\left(F_{X}(x)\right)-c_{1} x\right| \leqslant \varepsilon$, and therefore, as $\varepsilon>0$ is arbitrary, $\lim _{x \rightarrow+\infty}\left|F_{Y}^{-1}\left(F_{X}(x)\right)-c_{1} x\right|=0$, completing the proof.

REMARK 3.5. Theorems 3.4 and 3.5 were proved under the assumption $\left(\lambda_{1}, \lambda_{2}\right) \prec\left(\theta_{1}, \theta_{2}\right)$ on the hazard rates. Similar to what was mentioned with respect to Corollary 3.1, the conclusions of all these results still hold under the alternative assumptions $\lambda_{1}<\lambda_{2}$ and $\lambda_{2} / \lambda_{1}>\theta_{2} / \theta_{1}$.

\section{REFERENCES}

[1] I. Arab and P. E. Oliveira, Iterated failure rate monotonicity and ordering relations within gamma and Weibull distributions, Probab. Engrg. Inform. Sci. 33 (2019), 64-80.

[2] I. Arab and P. E. Oliveira, Iterated failure rate monotonicity and ordering relations within gamma and Weibull distributions_Corrigendum, Probab. Engrg. Inform. Sci. 32 (2018), 640641.

[3] I. Arab, M. Hadjikyriakou and P.E. Oliveira, Failure rate properties of parallel systems, Adv. Appl. Probab. 52 (2020), 563-587.

[4] R.E. Barlow and F. Proschan, Statistical Theory of Reliability and Life Testing: Probability Models, Holt, Rinehart and Winston, New York, 1975.

[5] N. Cai, W. Ni and C. Li, Some ordering properties of series and parallel systems with dependent component lifetimes, Comm. Statist. 48 (2019), 4764-4779.

[6] J. V. Deshpande, S. C. Kochar and H. Singh, Aspects of positive ageing, J. Appl. Probab. 23 (1986), 748-758.

[7] A. H. El-Bassiouny, On testing exponentiality against IFRA alternatives, Appl. Math. Comput. 146 (2003), 445-453.

[8] E. Fagiuoli and F. Pellerey, New partial orderings and applications, Naval Res. Logistics 40 (1993), 829-842.

[9] S. Kayal, Stochastic comparisons of series and parallel systems with Kumaraswamy-G distributed components, Amer. J. Math. Management Sci. 38 (2019), 1-22,

[10] S. C. Kochar and D. D. Wiens, Partial orderings of life distributions with respect to their ageing properties, Naval Res. Logistics 34 (1987), 823-829.

[11] S. C. Kochar and M. Xu, Comparisons of parallel systems according to the convex transform order, J. Appl. Probab. 46 (2009), 342-352.

[12] S. C. Kochar and M. Xu, On the skewness of order statistics in multiple-outlier models, J. Appl. Probab. 48 (2011), 271-284.

[13] C. Li and X. Li, Stochastic comparisons of parallel and series systems of dependent components equipped with starting devices, Comm. Statist. 48 (2019), 694-708.

[14] A. W. Marshall and I. Olkin, Inequalities: Theory of Majorization and Its Application, Academic Press, New York, 1979. 
[15] A. K. Nanda, N. K. Hazra, D. K. Al-Mutairi and M. E. Ghitany, On some generalized ageing orderings, Comm. Statist. 46 (2017), 5273-5291.

[16] J. K. Patel, Hazard rate and other classifications of distributions, in: Encyclopedia in Statistical Sciences 3, Wiley, 1983, 590-594.

[17] D. Sengupta, Another look at the moment bounds on reliability, J. Appl. Probab. 31 (1994), 777-787.

[18] S. Shaked and J. G. Shanthikumar, Stochastic Orders, Springer, New York, 2007.

[19] H. Singh, On partial orderings, Naval Res. Logistics 36 (1989), 103-110.

[20] T. Tossavainen, The lost cousin of the fundamental theorem of algebra, Math. Magazine 80 (2007), 290-294.

[21] W. R. van Zwet, Convex transformations of random variables, MC Tracts 7, Amsterdam, 1964.

[22] J. Wua, M. Wanga and X. Li, Convex transform order of the maximum of independent Weibull random variables, Statist. Probab. Lett. 156 (2020), 1-6.

[23] Y. Zhang, X. Cai, P. Zhao and H. Wang, Stochastic comparisons of parallel and series systems with heterogeneous resilience-scaled components, Statistics 53 (2019), 126-147.

Idir Arab, Paulo Eduardo Oliveira

CMUC, Department of Mathematics

University of Coimbra

Coimbra, Portugal

E-mail: idir.bhh@gmail.com

paulo@mat.uc.pt
Milto Hadjikyriakou University of Central Lancashire Larnaka, Cyprus E-mail: MHadjikyriakou@uclan.ac.uk

Received 22.12.2019;

revised version 15.2.2021 